\title{
Retraction Note to: Viscoelastic Behavior and Mechanical Properties of Polypropylene/Nano-Calcium Carbonate Nanocomposites Modified by a Coupling Agent
}

Mustafa Abu Ghalia ${ }^{1}$ Ibrahim Inuwa ${ }^{2}$ Azman Hassan ${ }^{2}$ Yaser Dahman $^{* 1}$
${ }^{1}$ Department of Chemical Engineering, Ryerson University, 350 Victoria St, Toronto, Ontario M5B 2K3, Canada

${ }^{2}$ Polymer Engineering Department, Faculty of Chemical Engineering, Universiti Teknologi Malaysia 81310 Skudai, Johor Bahr, Malaysia

Retraction Note: The Editor-in-Chief has retracted this article [1] following an investigation by Ryerson University. Concerns were raised regarding Figure 13C. It appears that parts of Figure 13C overlap with Figure 13B. As a result of the University's inquiry into this matter, it was determined that the research in question underlying the publication was not undertaken within Ryerson labs and a copy of the data was not retained by the authors. As the authors were not able to produce a copy of the underlying data for independent verification, they have agreed to retract the article. Mustafa Abu Ghalia, Ibrahim Inuwa, Azman Hassan, and Yaser Dahman agree to this retraction.

[1] Mustafa Abu Ghalia ${ }^{1}$, Ibrahim Inuwa ${ }^{2}$, Azman Hassan ${ }^{2}$, and Yaser Dahman*1, Viscoelastic Behavior and Mechanical Properties of Polypropylene/Nano-Calcium Carbonate Nanocomposites Modified by a Coupling Agent. Macromol. Res. (2016). https:// doi.org/10.1007/s13233-016-4083-8.

The online version of the original article can be found at https://doi.org/

10.1007/s13233-016-4083-8

*Corresponding Author: Yaser Dahman (ydahman@ryerson.ca) 